\title{
Metabolic correction therapy: Changing the healthcare management paradigm
}

\author{
Jorge R Miranda-Massari ${ }^{1,3}$, Michael J Gonzalez ${ }^{2,3 *}$, Jorge Duconge ${ }^{1,3}$, Miguel J Berdiel ${ }^{3}$, Ines Alfaro ${ }^{3}$ and Grace Kazuko $^{3}$ \\ ${ }^{1}$ School of Pharmacy, Medical Sciences Campus, University of Puerto Rico, San Juan, Puerto Rico \\ ${ }^{2}$ School of Public Health, Medical Sciences Campus, University of Puerto Rico, San Juan, Puerto Rico \\ ${ }^{3}$ Metabolic Correction Institute, Medical Sciences Campus, University of Puerto Rico, San Juan, Puerto Rico
}

The expenditure per capita on healthcare in the US is by far the highest in the world, but its effect on life expectancy and several other health outcome measures are not among the top 20 nations [1,2]. The high level use of medication and technology in the US raises health costs but this increased expense is not enough to produce the desired health outcome. The reason is simple; the model is not addressing the underlying causes of disease.

Modern society has created an environment that creates an array of health risks factors that are responsible for the increasingly high prevalence of chronic degenerative diseases. Low nutrient density food; loaded with colorants, preservatives, environmental toxins and the over-utilization of medication eventually cause depletion of necessary micronutrients in the body. This negatively affects the function of metabolic enzymes that control all physiological processes causing significant biochemical disturbances that are responsible for cellular dysfunction and damage. This problem results in a slower and inefficient detoxification process leading to intoxication, reduced immune system activity, unbalanced body microbiota, variations of hormonal balance and a decreased mitochondrial function. All these alterations eventually lead to one or more chronic degenerative conditions depending on the combination of the individual genetic background and their interaction with external risk factors.

Metabolic Correction proposes a structured model for efficient healthcare. The most cost effective way of improving health is providing quality and quantity of nutrients required while avoiding harmful compounds and facilitating their elimination. Metabolic correction is a concept that supports health maintenance and promotes the healing processes in an effective and safe way by improving the body's biochemical-physiological mechanisms. This is done by helping activate the metabolic enzymes necessary to facilitate relevant physiological pathways. Specialized individualized testing will identify the underlying metabolic causes of chronic illness, and Metabolic Correction will provide the necessary coenzymes and cofactors to balance metabolism, neutralize and eliminate offending agents while improving physiological functions.

Metabolic Correction Therapy is a tool for physicians to structure and monitor patient care and management. A profile of a patient's diagnostic laboratory testing variation can guide the selection of drugs, supplements and Dietary guidelines that minimize harmful side effects and ensure a more successful outcome. It can also indicate susceptibility to certain diseases before they become manifest, allowing the physician and patient to set out a plan for monitoring and preventing the condition by studying their metabolic pathways.
Metabolic Correction Characteristics:

- Preventive

- Coordinated

- Evidence-based

- Optimizes physicians treatment decisions making process

- Proactive and participatory, engaging patients in lifestyle choices

- Active health maintenance to compensate for genetic susceptibilities

Disease results of a deterioration of normal physiology that occurs when a particular organism (with its unique set of genes and metabolism) is exposed to an unfavorable environment. More specifically, health or disease will manifest itself by the interaction of nutrition, lifestyle and the influence of environmental contaminants that ranges from hydrocarbons, heavy metals to EMF radiation and even medications. Therefore in the $21^{\text {st }}$ century, a complete clinical assessment to pinpoint these particular issues is needed. This will require a complete detailed medical history, physical examination and comprehensive laboratory testing that should include whole genome sequencing, inflammation markers, immune testing, nutrient levels, heavy metal toxicity, xenobiotics, food intolerance/sensitivities and other necessary metabolic panels.

Medical research and education has established how multiple chronic diseases arise from lack or unbalanced nutritional factors and a toxic environment and yet the medical guidelines are mainly directed toward the use of synthetic compounds to alter our response to these conditions. In summary we are only treating the symptoms. Instead we should address the underlying causes of the disease. It is important to remember that deficiencies, insufficiencies, allergies, sensitivities, intolerances, toxicities and biotic imbalances can all be causes of disease. The therapeutic use of nutrition and scientific supplementation as explained by the concept of Metabolic Correction needs serious consideration by our health system as our first line of defense.

Correspondence to: Michael J Gonzalez, School of Public Health, Medical Sciences Campus, University of Puerto Rico, San Juan, Puerto Rico, Tel: 787758-2525; 787- 758-1405; Fax: 787-759-6719; E-mail: michael.gonzalez5@upr.edu

Received: February 13, 2016; Accepted: March 11, 2016; Published: March 14, 2016 
The increasing cost of medication and related morbidity and mortality in ambulatory patients in the USA has been documented in various studies. This cost increased from 76 billion dollars in 1995 to $\$ 177$ billion in 2001 [3,4]. At this rate the medication related morbidity and mortality is estimated to surpass 700 billion dollars in 2015. Medication induced morbidity and mortality is also related to its adverse effects. Some of which are an extension of the pharmacologic effect, such as allergies, some are related to the direct toxic effects of the drug or its metabolites and some of are related to the drugs nutrient depletion effect.

Studies in pharmaco-economics have postulated Pharmaceutical Care as a method to improve health care and reduce cost [5]. However, Pharmaceutical Care models as proposed so far do not include the concept of Metabolic Correction. Metabolic Correction is the use of a synergistic combination of micronutrients and cofactors in the proper active forms and doses that maximize the function of metabolic enzymes in most individuals [6,7]. Metabolic Correction actually compensates for drug induced nutrient depletion as well as for the nutrient depletion due to environmental toxins.

Metabolic Correction helps improve or rectify biochemical disturbances associated with a diversity of dysfunctional biological mechanisms that disrupt normal physiological processes leading to degenerative diseases. Metabolic Correction is the fine tuning of the cellular biochemistry by means of specific nutritional supplementation with the goal of improving cellular, tissue, organ, system and organism function [6].

Utilizing published data and a model of Metabolic Correction, we estimated the costs of treatment of diabetes and its complications in Puerto Rico and the impact of Metabolic Correction reducing costs. These estimates have been submitted for publication and are currently on peer review. In summary the costs reductions calculated for use in
$50 \%$ of population in this analysis were As follows: 1 . Costs reduction due to prevention of chronic complications of diabetes and prediabetes is \$ 63.8 million, 2. Cost reductions due adverse drug effects \$ 148.7 million and 3. Cost reduction due to loss of productivity $\$ 731$ million, for a total savings of $\$ 943.5$ million. This estimate of cost reduction was made by applying Metabolic Correction to only $50 \%$ of the population with diabetes. It is important to recognize that this model is not considering the savings due to reduction of hospitalization or due to reduced medication costs. Since that manuscript was submitted, we conducted yet another clinical study with 50 diabetic patients to determine the actual cost reducing effects of Metabolic Correction due to reduction in the cost of medication. The results of that study demonstrated that cost reductions in medication are very significant and in fact greater than anticipated. In conclusion, the application of the Metabolic Correction therapeutic approach seems to significantly reduce healthcare cost while improving quality of life of the patients.

\section{References}

1. Organization for Economic Co-operation and Development (OECD) (2015) Health ataGlance.

2. World Health Organization (2015) USA.

3. Johnson JA, Bootman JL (1995) Drug-related morbidity and mortality. A cost-of-illness model. Arch Intern Med 155: 1949-1956. [Crossref]

4. Ernst FR, Grizzle AJ (2001) Drug-related morbidity and mortality: updating the costof-illness model. J Am Pharm Assoc (Wash) 41: 192-199.[Crossref]

5. Isetts BJ (2012) Pharmaceutical care, MTM, \& payment: the past, present, \& future. Ann Pharmacother 46: S47-56.[Crossref]

6. González MJ, Miranda-Massari JR, Duconge J, Allende-Vigo MZ, Jiménez-Ramírez FJ, et al. (2015) Metabolic correction: a functional biochemical mechanism against disease-Part 1: concept and historical background. P R Health Sci J. 34:3-8.

7. Miranda-Massari JR, González MJ, Duconge J, Allende-Vigo MZ, Jiménez-Ramírez FJ, et al. (2015) Metabolic correction: a functional biochemical mechanism against disease-Part 2: mechanisms and benefits. P R Health Sci J. 34:9-13.

Copyright: (C2016 Miranda-Massari JR. This is an open-access article distributed under the terms of the Creative Commons Attribution License, which permits unrestricted use, distribution, and reproduction in any medium, provided the original author and source are credited. 